\title{
Imperativos \\ urbanos para el crecimiento económico y su planeación en la frontera noreste de México
}

\author{
Humberto Palomares León*
}

Abstract

One of the issues of the development as a goal is the search of those elements that enable to properly understand and link the geographical growth of the city for its eventual planning. The future planning, not only as an economical activity and urban services container, but also as a shaping tool, can be defined as the previous comprehension of the different actors' actions in a horizontal community organization, as well as the appropriate public differentiation of the expected results of those actions. The paper highlights the pertinence of locating the different effects of economy on territory, not as something geographically determined, but as a result of changing circumstances in public and private spaces for decision; spaces that can be noted as urban imperatives.

Keywords: urban imperatives, urban infrastructure, local planning, urban change.

*El Colegio de la Frontera Norte, correo-e: hpleon@colef.mx.

Economía, Sociedad y Territorio, vol. II, núm. 8, 2000, 687-719. 


\section{Resumen}

Una de las problemáticas del desarrollo como finalidad es la búsqueda de los elementos que permitan entender y conjugar, de manera adecuada, el fenómeno de la expansión física de la mancha urbana en las ciudades para su eventual planeación. Como herramienta moldeadora y no sólo de contención de la expansión de actividades económicas y servicios urbanos, la planeación del futuro puede definirse como el previo entendimiento de las acciones que toman los diversos actores en una organización comunitaria horizontal, y como la diferenciación pertinente y pública de los efectos esperados de dichas acciones. En el documento se llama la atención sobre la pertinencia de ubicar los efectos diferenciados que la economía forma en el territorio, no como algo predeterminado geográficamente, sino como producto de circunstancias modificables en los espacios públicos y privados de decisión que pueden ser observados como imperativos urbanos.

Palabras clave: imperativos urbanos, infraestructura urbana, planeación local, cambio urbano.

\section{Introducción}

La boga de la globalización económica ha encontrado eco en todos los aspectos que rodean al proceso de producción, distribución y consumo de los bienes y servicios. Sin embargo, pocas son las referencias académicas que se hacen al papel del espacio socialmente construido, como si los tiempos de producción hubieran sido modificados sólo para el just in time como sinónimo de ahorro de costos, de eficiencia y de monitoreo de mercados. La proliferación de estrategias productivas diversas que empujan a la configuración de espacios económicos diferenciados es, aunque escasamente reconocido, el imperativo más importante en nuestros días. Este proceso de reestructuración intraurbana ha sido poco explorado y menos aún explicado.

La cuestión urbana, como problemática específica del desarrollo económico desde la perspectiva académica marxista y neomarxista, surge con los trabajos de Manuel Castells (1974), Henri Lefebvre (1991 y 1996) y David Harvey (1985), entre los más reconocidos. La cuestión urbana es evidentemente crítica, su importancia radica en que, metodológicamente, va más allá de la descripción y diseño de la ciudad, más allá del acomodo mismo 
de los espacios y los usos, y se dirige hacia la exploración de los imperativos que el empuje económico y poblacional efectúa en las diferentes naturalezas de los territorios, entretejiendo lazos complejos y simples de estructuración intra e interurbana como resultado de la combinación de factores de desempeño económico y de organización socioproductiva intraurbana e intrarregional, estableciendo lo que denominamos imperativos urbanos.

A diferencia de los imperativos del desarrollo social humano (subsistencia, protección, afecto, entendimiento, participación, ocio, creación, identidad y libertad) y de los imperativos económicos (producción, distribución y consumo), los imperativos urbanos son específicamente resultado de decisiones en las que se han combinado los recursos naturales del territorio intervenido y la capacidad de confluir de los actores que participan en el proceso económico elaborando y descubriendo las ventajas de la localidad en términos intraurbanos (acuerdos entre actores del desarrollo social, político y económico para, cotidianamente, funcionar como ciudad) y en términos interurbanos (organización socioproductiva de los actores de la localidad para efectuar los arreglos económicos, financieros y de política necesarios para aprovechar las ventajas específicas con que cuentan: la distancia, recursos naturales, medios de comunicación, etcétera).

Uno de los propósitos principales de este escrito es iniciar el debate teórico sobre las implicaciones urbanas que tiene la expansión de actividades económicas y de la población en las ciudades, con relación a los esfuerzos por planificarlas. Es preciso volver la vista a los enfoques recientes de la urbanización y considerarla como un proceso con particularidades espaciales en cada localidad, de acuerdo con los recursos de acción de que disponen y con sus vínculos con las escalas de decisión, en el sentido económico de una multiplicación mayor de los flujos comerciales y en el sentido público de las posibilidades de acción de los gobiernos. El presente ensayo se apoya en la identificación de factores modeladores de cambio urbano, factores que cotidianamente ordenan, en algún sentido, el uso de los recursos cercanos, de los recursos del hinterland. Juegan papel importante las decisiones locales para el fomento de infraestructura y la distancia, aprovechada en el sentido del conocimiento del mercado y la desconcentración en el uso de los recursos disponibles.

La intensión es documentar la significativa influencia de elementos de la economía regional que generalmente se agregan y que mostrando especificidades pueden convertirse en herra- 
mientas integrales de análisis para la planeación: ¿qué relevancia tienen las condiciones diferenciales que se presentan en las localidades?, ¿cuáles de los elementos de naturaleza económica pueden catalogarse como parte indisoluble de la naturaleza urbana en ellas?, ¿cuáles son los elementos a ser considerados como componentes cualitativos de la planeación y que darían forma a lo que denominamos imperativos urbanos?

Esta reflexión se presenta en tres apartados. En el primer apartado se discute la pertinencia teórica de la economía urbana que ampara la necesidad de considerar a los imperativos urbanos más allá de los de naturaleza económica y de desarrollo humano. En el segundo apartado se presentan algunas hipótesis y consideraciones empíricas que alimentan la necesidad de pensar en los imperativos urbanos en términos de las condiciones que presenta la infraestructura de desempeño local, en el sentido más completo del término con información de las entidades fronterizas. ${ }^{1}$ Aquí también se discuten las deficiencias en la estructura local para la planeación y su desfase de los imperativos urbanos a que empujan los nuevos procesos productivos, establecidos de modo específico en la frontera noreste mexicana, que implican ciertas condiciones urbanas para su desempeño, desdeñadas por los imperativos económicos. El cuerpo de este apartado concluye argumentando la importancia de las condiciones de decisión como imperativos urbanos manejables a la escala de la planeación e insuficientemente explorados por las instancias de toma de decisiones. El supuesto básico es que las ciudades son lo que son por la combinación en el uso de recursos cuya fuente principal es la estructuración de decisiones corporativas o individuales conscientes, aprovechando las ventajas que les ofrecen la escala estatal y federal. En las conclusiones se sintetiza la estructura del trabajo y se presentan otros elementos de análisis susceptibles de ser incorporados como imperativos urbanos para el desempeño integral en las ciudades.

\section{Imperativos territoriales. Argumentación teórica en torno a la cuestión urbana}

En la literatura especializada sobre cuestiones urbanas se han desarrollado ciertos paradigmas del desarrollo que han privile-

\footnotetext{
${ }^{1}$ Por ejemplo, shelter, es un concepto que va más allá de la vivienda y se enriquece pensándolo en términos de protección humana y de apoyo para el eficiente desempeño económico y social de los habitantes de la misma (Pugh, 1997).
} 
giado los aspectos agregados de la problemática socioeconómica, y han tocado de modo superficial los aspectos que podrían darle un carácter particular a las regiones. La presión global de redes financieras no sólo ha aumentado los flujos de intercambio, también ha contribuido a delimitar los alcances regionales que tienen las áreas de desempeño económico diferenciado al interior de los países e incluso en términos de las relaciones territoriales entre ellos.

Los adjetivos de megaciudad, megalópolis, entre otros, son sólo algunos dentro de la literatura urbana delimitada a los aspectos macro del fenómeno y específicamente aquellos que ocurren en el ámbito de las relaciones ciudad-ciudad. Es un hecho cada vez más palpable que el tradicional imperativo territorial sintetizado en la relación campo-ciudad está siendo tendencialmente sustituido por infinidad de redes de intercambio campociudad-campo (Muheim y Freshwather, 1999). Estos fenómenos originados por el empuje económico están configurando variadas e interdependientes relaciones entre las áreas consideradas urbanas y rurales, entre las rurales y entre las urbanas.

Se han establecido un sinnúmero de procedimientos para establecer el orden en el cual se "organiza" el espectro urbano: regla, rango, tamaño, centralidad, nodalidad, etc. Sin demeritar lo conveniente del uso de técnicas para establecer las escalas del crecimiento urbano y su organización en un "sistema de ciudades", es conveniente desempolvar algunos argumentos no propiamente basados en el uso de técnicas de análisis, sino en el modo en el que las ciudades que ahora son, fueron.

La introducción del concepto imperativo urbano intenta darle un contenido más allá de los aspectos propios de la dinámica económica. Es un hecho que las inversiones en actividades productivas modifican el entorno de cualquier territorio, pero lo que también es necesario destacar es que esas decisiones y actividades se fortalecen o no, se asientan o no, en grado más o menos importante a partir del hinterland. Este es uno de los principales argumentos que deseamos desarrollar en este trabajo.

El paradigma dependentista está virando hacia el necesario desarrollo de ciudades medianas y su consecuente aportación a la innovación de procesos de desarrollo e intercambio comercial en la red global. En varios estudios se ha venido documentando el cambio hacia la desconcentración territorial de la población y las actividades económicas en México (Dehgahn y Vargas, 1999; Aguilar y Rodríguez, 1995; Aguilar et al., 1996). Los argumentos 
más importantes de los estudios que muestran dicha tendencia es el cambio de economías de escala a deseconomías de escala en las grandes metrópolis, que no es otra cosa que el agotamiento de los beneficios de la aglomeración y la pérdida de la eficiencia económica. La dispersión de la población y las actividades económicas en ciudades intermedias es la respuesta de tal agotamiento: literalmente, la población en México de 1950 al 2000 ha marchado desde el Centro-Sur hacia el Bajío-Occidente-Norte.

Sin embargo, esos son los argumentos económicos; pero los lugares en donde se desconcentró la actividad económica y la población no fueron cualesquier punto en el territorio. Son lugares donde la decisión local pudo haber jugado un papel crucial, donde las condiciones del hinterland han sido ampliamente desarrolladas y se ha hecho un uso combinado de recursos con mayor eficiencia para recibir el empuje económico. ¿Por qué se abandonan los territorios del sur-sureste ampliamente reconocidos por sus abundantes recursos y su clima principalmente templado, y se incrementa la población en las ciudades relativamente áridas del norte? ${ }^{2} \mathrm{La}$ información que ofrecen dichos estudios nos permite suponer que probablemente haya un tamaño adecuado de ciudad, donde se mantiene la eficiencia urbana (que puede definirse como las condiciones de infraestructura para el desempeño económico); pero también supondría que existen elementos adicionales, territoriales, que convertidos en particularidades de las ciudades ofrecen funcionalidad productiva (que puede considerarse como el marco u organización territorial de los actores) de la que emanan disposiciones y códigos propios.

Es obvio que existen argumentos mas allá de los imperativos económicos de la escasez de los recursos; es obvio que el modo en el cual se organiza la población para tomar decisiones está jugando un papel significativo y que eventualmente tiene un peso mayor que los clásicos imperativos económicos. Es probable que una de las respuestas tenga que ver con el modo en el cual se esté aprovechando la distancia entre ciudades con infraestructura semejante y que en realidad no haya competencia, sino complementación al desarrollar política pública y planeación urbana (Rohe, Adams y Arcury, 2001).

2 En Dehggan y Vargas (1999) se muestran para tres años, 1950, 1970 y 1990, mapas de la distribución de las ciudades en los que se aprecia la desconcentración de la población. Ciudades millonarias en el 2000 como Juárez y Tijuana, en el norte, se mantuvieron en su tamaño de población, menor de 500 mil, de 1950 a 1970 . Rangos de tamaño de población entre medio millón y el millón, inexistentes en 1950 se han multiplicado en 1990. 
El complemento ineludible de estas reflexiones se relaciona con la pertinencia de la planeación a escala local. La planeación a escala urbana, inaugurada hace más de un siglo por Howard y cuyos propósitos universales fueron la combinación de las ventajas de lo urbano y lo rural (Hall, 1992) ha sobrevivido sólo en el aspecto de la forma de la ciudad y ha estado ausente el aspecto social y económico. En México se cuenta con una larga tradición de planeación (véase Garza, 1996); sin embargo ha estado condicionada a los vaivenes que ha venido estableciendo el paradigma de desarrollo a escala federal. A nivel local son pocos los gobiernos que han empezado a manejar fondos suficientes y estrategias propias para efectuar planeación local (Cabrero, 1996; Cabrero et al., 1996). Empero, la estrategia del manejo de la problemática local a escala local está reproduciendo los esquemas verticales de decisión y no se han estudiado las ventajas en términos de los esquemas horizontales: puede considerarse que estamos aún en la planeación del pasado.

De acuerdo con Castells (1974), la organización intraurbana o estructura social determinaba las posibilidades de la planeación y de modo específico dependía “...directamente del estado de la politica, es decir, de la presión social ejercida por la fuerza del trabajo". ${ }^{3}$ Quizá la planeación en el ámbito urbano ha sido rebasada por la presión social que ejerce la fuerza de trabajo (al establecerse en la periferia de las áreas urbanas), pero en mayor medida ha sido rebasada por el modo en el cual se resuelve, se dirime, la contradicción entre quienes son dueños de los recursos locales y quienes, a partir de la estructuración social -organismos públicos y privados- requieren hacer uso de dichos recursos explotables del hinterland. La apertura en el ámbito público local, del modo en el cual se resuelve esta contradicción podría ofrecer mayores elementos de planeación urbano-comunitaria. Si se tuviera el conocimiento de las alianzas de clase para empujar hacia tal o cual conflicto de intereses en el ámbito urbano, es probable que fueran superadas en un esquema de planeación local. Más aún, es probable que el desconocimiento de estas circunstancias propicie la inoperancia de los planes, incluso aquellos con instrumentos novedosos o ampliamente discutidos en la arena pública.

\footnotetext{
${ }^{3}$ En La cuestión urbana de Manuel Castells se manejan algunas hipótesis para el estudio de la problemática urbana. La relacionada con la planeación o planificación consiste en superar dos contradicciones antagónicas básicas del sistema capitalista: la contradicción entre fuerza de trabajo y no-trabajo y la contradicción entre quienes se apropian y quienes son dueños de las fuerzas productivas.
} 
De este modo se considera que los imperativos urbanos son el resultado de condiciones esencialmente diferenciales entre los territorios, aunque exista un patrón de desempeño económico relativamente homogéneo, por ejemplo, los considerados modelos de industrialización o las actividades económicas predominantes, etcétera. La aproximación al estudio de los imperativos urbanos se realiza bajo tres condiciones de desempeño urbano: la infraestructura intraurbana como modeladora de cambios, el diseño de la planeación y algunas circunstancias de toma de decisión para el uso de los recursos locales.

\section{Aproximación empírica al estudio de los imperativos urbanos en el noreste de México}

Los imperativos económicos generalmente inducen el florecimiento de medianas y grandes ciudades. Es probable que el florecimiento y decaimiento de las primeras civilizaciones haya dependido más de las condicionantes económicas que de una predeterminación o acuerdo político en las comunidades. El decaimiento de las grandes civilizaciones del pasado aún es poco claro, y la probable explicación de dicho suceso se ha relacionado con la falta de las condiciones económicas (población y recursos) para su continuación. Sin embargo, el decaimiento de ciudades modernas en Europa y los Estados Unidos no ha significado su desaparición, sino un estatus diferente al que jugaron cuando fueron puntas del crecimiento económico (Storper y Walker, 1989).

El diferente estatus que en el transcurso del tiempo tienen las áreas urbanas puede ser analizado a partir de la combinación de tres aspectos de su desempeño: en primer lugar, ¿̇en qué medida la infraestructura modela los cambios dentro de cualesquier área urbana, tanto para alojar nuevas y mayores actividades económicas y población, como para modificar su estatus dentro del sistema de ciudades en una región, país o el mundo? En segundo lugar, ¿qué importancia tiene la planeación a escala local y cómo se adecua a las condiciones de desempeño económico y cambio poblacional? Y por último, ¿cómo puede catalogarse la organización comunitaria en términos de las decisiones que se toman para utilizar los recursos del hinterland? 


\subsection{La infraestructura como elemento modelador del cambio urbano}

Una consideración hipotética general sobre la cobertura de infraestructura urbana es que la incorporación de los recursos naturales y poblacionales al mundo de la producción, distribución y consumo ejerce presión hacia dos fenómenos conocidos en las áreas urbanas: por una parte, hacia la concentración y centralización de las actividades económicas y sociales hacia ciertos y pocos puntos del territorio - no son cualesquier punto en el territorio, ya que presentan restricciones locativas. Y por otra parte, derivado del anterior, se ejerce presión para que dichos puntos desarrollen y respondan con eventos exclusivos ante la presión capitalista por generar excedentes; originando especificidades territoriales, cuya espacialidad no depende en sentido limitado del factor económico, pues se incorporan aspectos cualitativos igual de importantes como son las habilidades intergeneracionales, ${ }^{4}$ las características demográficas de los habitantes (población disponible para el trabajo), la cantidad y calidad del suelo susceptible de ser intervenido (extensión posible de la mancha urbana, vialidades y espacios para construcción y conservación ambiental, etcétera) y el ejercicio del poder político (en el manejo de mecanismos de aliento o desaliento de infraestructura para la eficiencia urbana), principalmente.

En este sentido, se sugieren dos condicionantes particulares. La primera delinea el desarrollo de dos procesos encontrados: por una parte, la consolidación relativa, espacial y temporal de la inversión y extensión capitalista, para la obtención de excedente a través de los mercados de producción globales ha traído consigo, como tendencia, la normativización de los estándares mínimos de vida de las sociedades, puesto que permite, como tendencia, que el mismo conjunto de mercancías esté disponible en todo el mundo (Boltvinik, 1996).

Sin embargo, la respuesta locativa depende de las especificidades que ofrece el territorio intervenido, con posibilidades de contrarrestar las tendencias globalizadoras. Para decirlo de otro modo: la política federal de generar espacios abiertos al comercio no sólo desalienta tendencias hacia espacios homogéneos sino que atomiza la producción a partir de la división territorial-espa-

\footnotetext{
${ }^{4}$ Referidas a las que adquiere la población de sus padres o abuelos. En otras palabras, las que transmiten éstos a su descendencia y que a su vez estos transmiten a la siguiente generación.
} 
cial del trabajo, como respuesta de los elementos adicionales a los económicos a que se hace alusión arriba. De manera específica, puede afirmarse que en el diseño y la aplicación del Programa de Industrialización Fronteriza (PIF) no se consideraron las implicaciones urbanas y sociales que se desenlazarían en mayor o menor medida en cada localidad considerada para el proyecto: migración, ocupación ilegal del territorio, encarecimiento del uso del suelo, etcétera, que pueden considerarse respuestas propias de dichos fenómenos dentro del territorio, y que probablemente no estaban incluidas como lógicas resultantes del PIF.

Una segunda consideración particular es que las entidades del norte del país se han visto modificadas en distinta forma al aumentar el empleo de actividades manufactureras, de servicios y comerciales. Esto ha significado la creencia, en términos relativos, que la situación de carencia económica de la población es menor en general que en el resto del país, ya que se presenta menor concentración del ingreso vía salario mínimo y menor proporción de pobreza (extrema y relativa) por ingreso, con respecto a la registrada a nivel nacional (Alegría, 1994; Camberos y Bracamontes, 1998). Sin embargo, el que en las últimas dos décadas (1970-1980 y 1980-1990) se hayan presentado en las entidades del norte de México importantes incrementos de actividades económicas y de población no significa que las condiciones de carencia se hayan reducido de modo general.

De hecho, en materia de infraestructura para la eficiencia urbana se considera que aún se observan condiciones diferencialmente paupérrimas. Con base en un ejercicio aplicado a la escala regional (Aguilar, Almaráz y Palomares, 1998) se puede afirmar que existe desigualdad en términos de los recursos de infraestructura regional de que disponen las comunidades, y es probable que esta situación responda a dinámicas fuera del alcance del crecimiento económico por sí mismo, y se incorpore a la esfera de la desigual calidad del empleo generado. Esto implica que la investigación tiene que ir más allá de la evaluación de las condiciones en las que se ha expandido la industrialización en el norte del país, pues si bien ha aumentado el empleo, otras circunstancias le han impreso condiciones de aumento en la carencia de infraestructura básica para el desempeño urbano del bienestar en localidades de economía dinámica, como es el caso de las localizadas en la franja fronteriza norte de México.

De 1930 a 1950 se presentó el crecimiento demográfico de mayor relevancia en las entidades fronterizas. Se ha estimado que 
mientras la población nacional se cuadruplicó de 1930 a 1980 , en las entidades fronterizas la población se multiplicó por 11 veces (Zenteno, 1993: 13). Sin embargo, la multiplicación no es generalizada ni homogénea en todas las entidades. En la gráfica 1 se observa que sólo Baja California y Tamaulipas mostraron tasas de crecimiento considerablemente mayores a la nacional hasta 1970; para el primer caso, está claro que el auge de la colonización fue primordial, ya que contaba con una población marginal y poco tiempo de haber dejado de ser territorio. Otra diferencia importante es que si bien el punto de inflexión de la tasa de crecimiento nacional se da alrededor de los años setenta, los estados de Baja California, Tamaulipas y Coahuila ya lo habían presentado en los años cincuenta, aunque la última entidad registró un nuevo auge dos décadas después. Las diferencias no acaban: a pesar de que las tasas de crecimiento demográfico en los estados fronterizos siguen siendo mayores que el promedio nacional, la tendencia es hacia la disminución, a excepción del estado de Coahuila.

\section{Gráfica 1}

Tasas de crecimiento de la población por periodos en los estados fronterizos del norte de México. 1895-1995

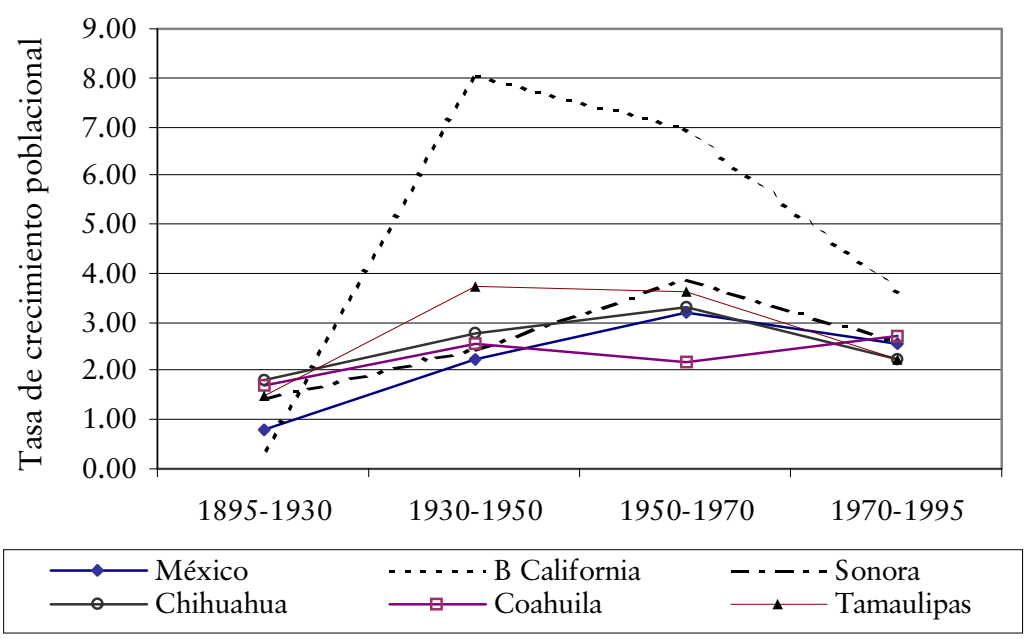

Fuente: Elaboración propia con base en INEGI, Censos de Población y Vivienda I, V, VII, IX y de los años 1895, 1930, 1950, 1970 e INEGI, Conteo de Población en 1995.

Debido su cualidad de ubicación como puntos de expansión manufacturera y de servicios, en las localidades fronterizas se ha extendido el tejido urbano y su paisaje ha dejado de ser 
visto como área sin dueño o sin ley. Se han incrementado las disposiciones y acuerdos binacionales cuyo principio básico no sólo es la expansión económica, sino también el cuidado de los recursos naturales para su permanencia. Estos acuerdos, empero, no han establecido condiciones particulares para situaciones diferenciadas a lo largo de la frontera, donde las tasas de expansión sociodemográfica se combinan y no tienen que ver con el tamaño actual ni con el perfil económico, sino con las posibilidades de administrar y empujar hacia el fomento de actividades con amplio valor agregado, y con efectos de arrastre que integren las cadenas locales de producción hacia un aprovechamiento mayor de recursos humanos altamente capacitados.

De los años setenta a la fecha han transcurrido dos fuertes crisis financieras que pusieron a prueba la estructura productiva nacional y regional. Tal como sucede en las épocas de auge y expansión, no todos los sectores quebraron ni el quiebre se presentó en todas las entidades. Aunque Baja California recibía constantemente población en edad laboral, pasó de representar cerca del dos por ciento del valor agregado nacional en 1980 a poco más de uno por ciento en 1990. La misma tendencia se presentó en Sonora: de representar casi 2.5 por ciento del valor agregado nacional en 1980, pasó a contribuir con 1.4 por ciento en 1990. Sin embargo, ambas entidades remontaron en los tres años siguientes para ubicarse con una participación de 2.5 por ciento en 1994. Hay que destacar que, en cuanto a valor agregado, Chihuahua y Coahuila mantuvieron e incrementaron sus participaciones más de dos por ciento; Coahuila llegó a 3.4 por ciento en 1990 a pesar de las crisis. Sólo en Tamaulipas se presentó una situación contrastante: aumenta en más de uno por ciento su participación de 1980 a 1985 , cae casi uno por ciento en los siguientes cinco años y vuelve a repuntar hasta situarse arriba de 2.5 por ciento en 1994 (véase la gráfica 2).

El comportamiento sectorial es también contrastante. En la gráfica 3 se muestran los cambios sectoriales en cada entidad fronteriza durante dos décadas. Baja California registra los rangos mínimos de cambio, aunque la mayoría son negativos, excepto la manufactura y los servicios que inician el repunte desde los años ochenta hasta la actualidad. Algo semejante ocurre en Sonora, salvo que los rangos en la pérdida de participación son mayores a los de Baja California y sólo aumenta su participación en servicios, manufactura y comercio hasta antes de los años ochenta. Sólo en Chihuahua se presentó un comportamiento constante del valor agregado a favor; todo lo contrario sucedió en Tamau- 
Gráfica 2

Cambio en la participación del valor agregado total por entidad fronteriza con relación al nacional

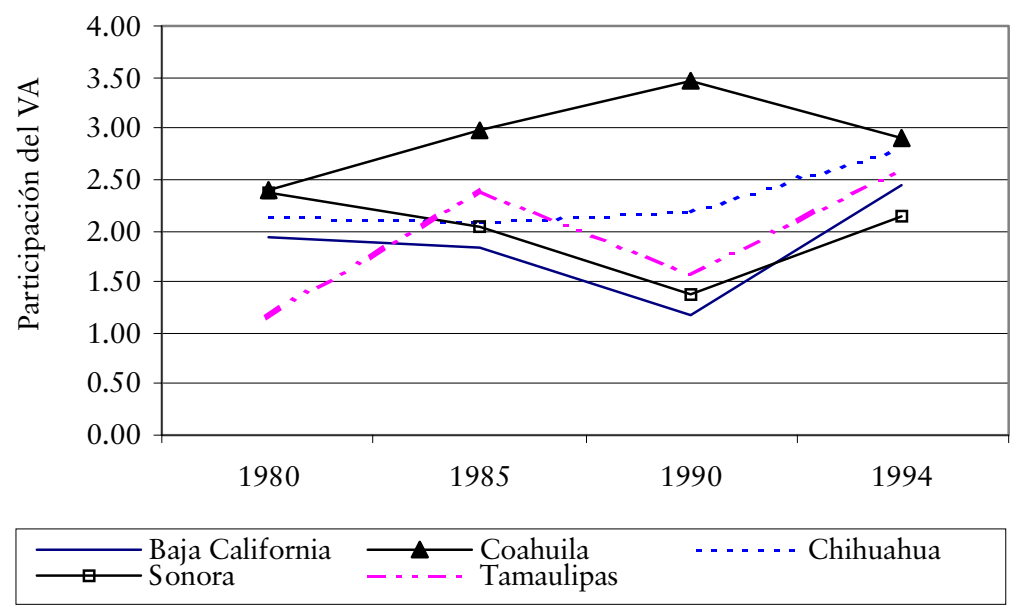

Fuente: Elaboración propia con base en INEGI, Censos Económicos 1980, 1985, 1989 y 1994.

\section{Gráfica 3}

Cambio en la participación porcentual del valor agregado en las entidades fronterizas del norte de México con relación al nacional

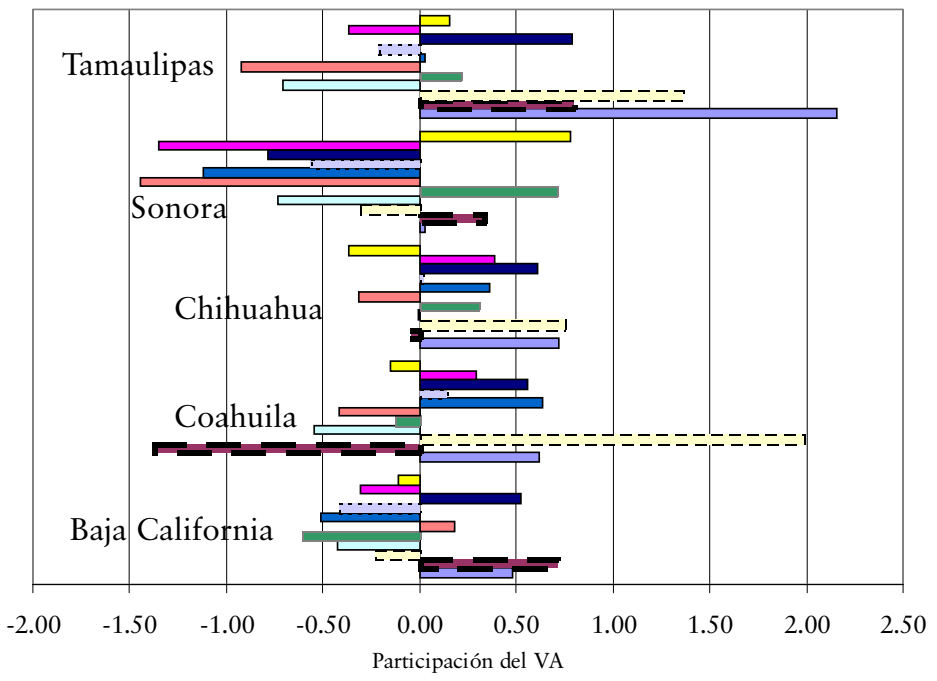
$\square$ Servicios, 75 a 85

Fuente: Elaboración propia con base en INEGI, Censos Comerciales, de Servicios e Industriales, 1975, 1980, 1985, 1990 y 1994. 
lipas y Coahuila, entidades que presentaron altibajos durante el periodo de crisis de la primera mitad de los años ochenta, aunque en general tuvieron un aumento en las actividades manufactureras y de servicios.

La información nos permite establecer tendencias en materia de imperativos urbanos. En primer lugar, la distancia es un elemento que continuará delimitando posibilidades de expansión económica. A pesar de que en el noroeste se localiza la mayor población relativa de la franja fronteriza, es probable que la expansión de la manufactura en Tijuana y Ciudad Juárez no genere el valor agregado suficiente para invertir en las condiciones mínimas de desempeño urbano; el sector servicios en estas ciudades sería sólo el complemento a tal expansión manufacturera, sin que se constituya como un sector con dinámica económica de arrastre equiparable a lo que sucede en el noreste, donde se presenta un uso intensivo del territorio y donde los servicios han llegado a constituir la palanca de expansión económica del tejido urbano. El imperativo de la extensión urbana como naturaleza productiva en el noroeste puede explicar el rezago en infraestructura; asimismo, el imperativo de la intensidad en el uso del espacio en el noreste puede explicar la imposibilidad de lograr mayor eficiencia urbana e impedir el aprovechamiento completo de los recursos locales para una efectiva funcionalidad productiva. Es un hecho que estas condiciones de expansión económica y demográfica implican todo un mosaico diferenciado, con lo que podemos asegurar que en las localidades fronterizas lo regional radica mucho más en la heterogeneidad productiva, y en menor medida en el hecho de ser frontera con los Estados Unidos.

Aunque han existido programas y planes relacionados con el impulso económico, la visión específica de ciudades ha estado al margen de los objetivos y metas. ¿Para qué se quiere aumentar el desempeño económico en una ciudad? Porque se considera que es la célula del desarrollo social. ¿Es cierto esto? ¿Los altos indicadores de desempeño económico han llevado a cubrir completamente el desarrollo social, entendido como la cobertura completa de las necesidades básicas de la población? Es probable que la rapidez con la que aumentan los flujos financieros y económicos esté empujando a nuevas dinámicas de desempeño urbano que estamos enfrentando con ideas de planeación ya superadas. 


\subsection{Desempeño urbano de futuro con planeación del pasado}

Resulta interesante observar que en las décadas de los setenta, los ochenta y los noventa, en México se presentaron fenómenos sociales que poco tenían que ver con los propósitos, la crítica y las agendas resultantes de las reuniones Habitat I (1976) y Habitat II -realizada dos décadas después- como fueron la desconcentración y reconcentración del ingreso nacional, el aumento y disminución del salario real que inauguró la inserción masiva del hogar nuclear y ampliado al mundo del trabajo, y el cambio en los patrones de edificación de vivienda de adobe al ladrillo y/o tabique (Boltvinik, 1994; Hernández, 1997) con la correspondiente minimización de los espacios para la convivencia y recreación en las áreas urbanas. Si bien se podía presumir del crecimiento de los empleos, era limitado hacer lo mismo con relación a la infraestructura para el acondicionamiento de la vivienda, que se encarecía y no guardaba relación alguna con el aprovechamiento de las economías de aglomeración que hicieron florecer las grandes metrópolis.

Varias líneas de trabajo y estudio han surgido luego de que se le reconoce al municipio la importancia que tiene a escala territorial. Existen perspectivas sobre el reparto de los recursos recaudados (la escala federalista), perspectivas sobre la eficacia y eficiencia de que son capaces los esquemas municipales de decisión (la escala del ejercicio público), así como los instrumentos de innovación que se han creado en algunas escalas gubernamentales. La planeación, por otra parte, ha sido objeto de reformulaciones y redefiniciones sobre el objeto y el cómo (la ejecución). Aunque se reconoce su importancia, en el mejor de los casos se ha limitado a la administración de ciertos recursos con finalidades determinadas o al acuerdo de ejecución de ciertas obras que modifican el espacio sin considerarlas como insumo de planeación.

Si bien ya no se planea desde la federación, las decisiones más relevantes siguen siendo tomadas a ese nivel. Por otra parte, las nuevas condiciones de desempeño económico han puesto a competir a los gobiernos locales por inversiones y recursos de infraestructura que les permita ofrecer amplias condiciones de localización e infraestructura de promoción a quienes toman ese tipo de decisiones. Eso ha significado planear para competir... y luego administrar. 
Evidencia de primera mano indica que existen pocas posibilidades de que la escala territorial del desarrollo municipal desempeñe los papeles deseados de promoción sin modificar cualitativamente el esquema de planeación. Se considera que el sustento teórico de la planeación estratégica y la planeación para competir $^{5}$ es una visión con modelos limitados al corto plazo, ya que es probable que en ciertos sectores se presenten problemas estratégicos, que pueden ser atendidos como condición primaria para la promoción de actividades, pero que serán resueltos en definitiva con una visión de planeación de largo alcance e incluyente socialmente.

Del mismo modo, pueden existir ciertos rasgos de la estructura productiva que pueden ser utilizados como gancho de promoción, siempre y cuando existan mecanismos de regulación que impidan la pérdida de manejo institucional. Resulta obvio que las necesidades de bajar las decisiones a un esquema horizontal no sólo dependen de contar con mayores recursos, sino con un aparato especializado que se conjugue con instancias de amplia participación pública e instrumentos de información constante y actualizada para la toma de decisiones.

A la posición escéptica de Herodoto sobre el discurso hacia las ciudades en el sentido de que "la felicidad humana nunca ha permanecido en un solo lugar" (citado en Storper y Walker, 1989) puede sugerirse el complemento de que el cambio de la infelicidad a la felicidad en ciudades preurbanas no siempre es posible en el sentido histórico, ya que se establece una especie de umbral del cambio en el que dichas localidades no acaban de madurar por circunstancias locativas específicas de organización socioeconómica, que inhibe o dificulta la esperada "felicidad" para la población residente. En este umbral, y con diversas facetas, se encuentran las localidades fronterizas del norte de México, que no acaban de madurar en la oferta de las condiciones urbanas mínimas que se ofrecen en las ciudades fronterizas del sur de EEUU, con las que comparten adyacencia.

Como resultado de su importante actividad económica y de la implementación de políticas económicas con claros (aunque limitadamente planeados) efectos regionales, las ciudades fronterizas han experimentado un crecimiento tanto poblacional como económico significativo en los últimos cuarenta años. Esta actividad económica ha implicado en su mayoría el crecimiento

\footnotetext{
${ }^{5}$ Donde se recoge el esquema neoclásico del costo-beneficio y la maximización de recursos escasos.
} 
de la mancha urbana que supera al observado en otras regiones y ciudades del interior del país. En vísperas del siguiente siglo, las localidades ubicadas cerca o en la frontera norte de México mantienen la ventaja comparativa de la distancia al mayor mercado de consumo como factor clave para el desempeño económico de los sectores exportadores y los mercados de trabajo. Del mismo modo, la población residente en dichas localidades enfrenta una serie de retos y problemáticas, derivados de esta dinámica, que requieren una solución adecuada. El reto pendiente es el logro de un desarrollo económico armónico y equilibrado con el crecimiento urbano ordenado y sustentable.

Para tal caso en la mayoría de las localidades urbanas de México el reto particular es la administración del crecimiento urbano bajo un esquema claro de planeación del desarrollo. El reto es grande, ya que asistimos a la planeación de ciudades del futuro, pero con esquemas del pasado. Los nuevos paradigmas tecnológicos y productivos avizoran cambios diferenciados en intensidad a escala territorial. Esto significa que en algunos casos pareciera que ciertas localidades cuentan con los recursos para potenciar el crecimiento de ciertas actividades económicas y resulta que pueden ser otras circunstancias las que determinen el cambio territorial de la inversión económica.

Resulta sorprendente que luego de medio siglo de experiencia y ejercicio de planeación permanezca el interés genérico de las finanzas gubernamentales (déficit y superávit comercial, balanza de pagos, etcétera) y la estructura productiva (inversión pública y privada, consumo público y privado, ocupación del territorio, etcétera) sobre las repercusiones que se generan a partir de la proyección de los volúmenes de inversión y las modalidades de financiamiento para las actividades productivas. En la primera línea se inscribe la planeación e investigación sobre el crecimiento industrial, el crecimiento urbano y el desenvolvimiento de la política pública en sus distintos niveles de toma de decisiones que se vienen presentando en el territorio. La segunda línea, aún no contemplada, implica la observación de las expectativas sobre los paradigmas no sujetos a control institucional como son la reestructuración industrial, la formación del mercado de trabajo y las condiciones sociales y de bienestar, derivadas de los efectos inducidos que han llegado a conformar dinámicas propias cuyos espacios intervenidos (familia, hogares, comunidad) debilitan el margen de manejo institucional de los problemas que se generan. 
El divorcio entre el pensar y actuar se relaciona con la diferencia entre el proyectar y ajustar. Esto último, que en apariencia implica ejecución de mecanismos y medidas para la planeación, es la parte correctiva de la visión futura del cambio. Salvo casos excepcionales, el ajuste de la proyección (objetivos generales y metas trazadas) no ha tenido cabida en los procesos de planeación, llevando a situaciones inmediatistas (no por ello cortoplacistas) que borran la huella del orden y progreso buscados con ahínco en la planeación institucionalizada. Aunque parezca contradictorio, la institucionalización de la planeación no ha llevado a que se institucionalice su práctica correctiva, ya que no hay garantía de seguimiento institucional de lo planeado, sólo del ejercicio en sí mismo, puesto que lo establecen las normas del ejercicio del gobierno, consignadas en leyes y reglamentos. Esto implica un doble problema: su limitado carácter enunciativo y su falta de legitimación social. El primero resulta de las limitaciones institucionales de la planeación vertical y centralista, así como de la precaria y poco confiable información con la que se diseña; el segundo es un reclamo que no ha acabado de nacer en los planes de desarrollo, necesario para el ajuste y corrección de la visión futura de los especialistas, pero sobre todo, por la creciente participación de actores no sujetos a control corporativo o gubernamental que juegan papeles imprescindibles en la nueva realidad socioeconómica. $^{6}$

Asociado a lo que se denomina teoría urbano-cultural, se han presentado estudios recientes sobre el cambio urbano que se produce al orientarse, en determinada ciudad, hacia nuevos patrones de consumo comunitario donde se ha mudado de la venta de un determinado imaginario urbano, en el cual cierta clase o grupo económico predomina, hacia la construcción del imaginario urbano a través de la expresión comunitaria en los medios de comunicación masivos (Greenberg, 2000). Estos nuevos paradigmas hacen pensar en esquemas de planeación flexible, pero sobre todo, esquemas de planeación que ofrezcan amplias posibilidades de intervención de agentes afectados o beneficiados, generando un claro esquema de gestión y negociación horizontales.

${ }^{6}$ Es reconocido por los especialistas de la OCDE que "las complejas relaciones entre la desigualdad socioeconómica y la exitosa acción colectiva para la construcción de instituciones de auto-gobierno en el ámbito local es todavía un área en economía desatendida por la investigación” (Bardhan, 1997: 59). Aun más, en el control de la estructura de gobierno debería considerarse a gente que cuenta con la información y los incentivos, y a quienes, al mismo tiempo, podrán cargar con la responsabilidad institucional por las consecuencias políticas y económicas de sus actos (Bardhan, 1997: 60). 
Ante estas nuevas circunstancias, se prosigue con los denominados planes maestros o directores de desarrollo urbano, cuya utilidad es poca o nula de acuerdo con los comentarios que han vertido los directores responsables de los aspectos urbanos de las localidades fronterizas, a quienes hemos entrevistado. Conviene traer a colación situaciones anecdóticas que fueron narradas por los directores del Departamento de Planeación en Reynosa y Matamoros. En entrevista realizada por separado, ambos coincidieron en que la propuesta federal de los nuevos usos de suelo programados (no planeados) para el futuro estaban fuera de las necesidades reales de uso del suelo en sus respectivas localidades. En varias ocasiones las autoridades tenían que aceptar ciertos cambios en el uso del suelo debido a que los constructores habían conseguido los permisos o hecho las gestiones (negociaciones) necesarias para llevar a cabo sus proyectos.

Las experiencias particulares de planeación en la escala local supone criterios heterogéneos, ya que aún depende de factores no controlados por la organización pública. De este modo, la diferenciación de los alcances de la planeación a escala local aún depende en gran medida de los recursos de las entidades y la federación. Sin embargo, en las respuestas locativas y los desenlaces territoriales han jugado un papel relevante la utilización de los recursos del hinterland, es decir, los recursos (humanos, naturales, mecanismos de organización, etcétera), relativamente cercanos al espacio urbano que es intervenido.

\subsection{Las decisiones locales en la utilización de recursos}

La tardía colonización del norte de México en el siglo XIX tuvo lugar, entre otras circunstancias, por la existencia de amplios espacios áridos (que permitían suponer la nula existencia de valles y lugares propicios para actividades de explotación de los recursos) y por una constante resistencia de las tribus a ceder espacios habitables. Nadie hubiera imaginado que lo que significó una ruptura política al instaurarse en 1848 la división más importante en la historia del noreste de México, se revertiría en un rico y complejo sistema de ciudades en ambos lados de la frontera. Tal vez el inicio de este sistema se deba a la existencia de asentamientos humanos que datan de finales del siglo XVI, ${ }^{7}$ sin embargo, es

\footnotetext{
${ }^{7}$ Extendidos a lo largo del Río Bravo (Río Grande para los estadounidenses) y cuya misión fue la colonización en la Jurisdicción del Nuevo Reyno (sic) de León (Monterrey, Villaldama, Sabinas, Lampazos y Vallecillo) y el Nuevo Santander (Laredo, Gue-
} 
posible también que se deba a la respuesta que han venido promoviendo, en una escala regional y local, los pobladores, manifestando sus intereses a través de sus organizaciones corporativas o a través de las instancias comunitarias del poder público.

Primero como un sitio de enlace para el exterior y el centro de la corona española, luego como límite entre México y los Estados Unidos, la ruta comercial del noreste (y la conformación urbana de sus asentamientos) cambia a medida que presiona la expansión de sus actividades económicas. Consideramos que el primer cambio urbano del noreste se observó a finales del siglo XIX en la particularidad de la expansión de la infraestructura para el comercio que promovió una diferenciación de la rutas y con ello del crecimiento urbano. ${ }^{8}$ Esta circunstancia diferenció las extensiones y los alcances territoriales de los procesos productivos ya que, en relación al noroeste, sólo en el noreste se intensificaron las vías de acceso comercial para el intercambio de mercancías, en relación con el de personas, hacia los Estados Unidos (véase el plano 1).

Se considera que el siguiente cambio urbano se establece hasta las décadas sesenta y setenta con el establecimiento de la industria maquiladora de exportación (IME), con una intensión clara de ofrecer, desde la visión centralista, a la franja fronteriza como un territorio homogéneo susceptible de ser intervenido por cualquier tipo de actividad manufacturera. Si bien estamos ante su consolidación, las particularidades locales han emergido en los asentamientos fronterizos: en algunos se ha acogido a la IME como la vanguardia de la expansión urbana, mientras que en otros sus efectos no tienen tal consideración y destaca una estructura económica que la combina con el establecimiento de industria extractiva y la industria para la generación de energía eléctrica. A diferencia de las ciudades de la frontera tamaulipeca, Piedras Negras se funda a mediados del siglo XIX a partir de una colonia militar, resultado de los tratados de Guadalupe en 1848. También a diferencia de aquellas ciudades, en Piedras Negras el cam-

rrero Viejo, Mier, Camargo y Reynosa). Para mayores detalles se puede consultar Robles (1979).

${ }^{8}$ En Alarcón (1990 y 1997) se destaca que la extensión del ferrocarril hasta Laredo en 1881 permitió el ajuste de la ruta comercial del noreste, ya que hasta entonces sólo consideraba los asentamientos de Monterrey, Camargo, Mier y Matamoros. Asimismo, se extiende hacia el sur en Saltillo y se conectó en 1888 con la ciudad capital. Hubo de esperarse, como luego sucedió, el aumento de ciertos flujos económicos que impulsaron el crecimiento espacial de Nuevo Laredo. Y con ello también, posibilidades e imposibilidades de administración de su crecimiento. 


\section{Plano 1 \\ Infraestructura disponible en ciudades fronterizas del norte de México. 1995}

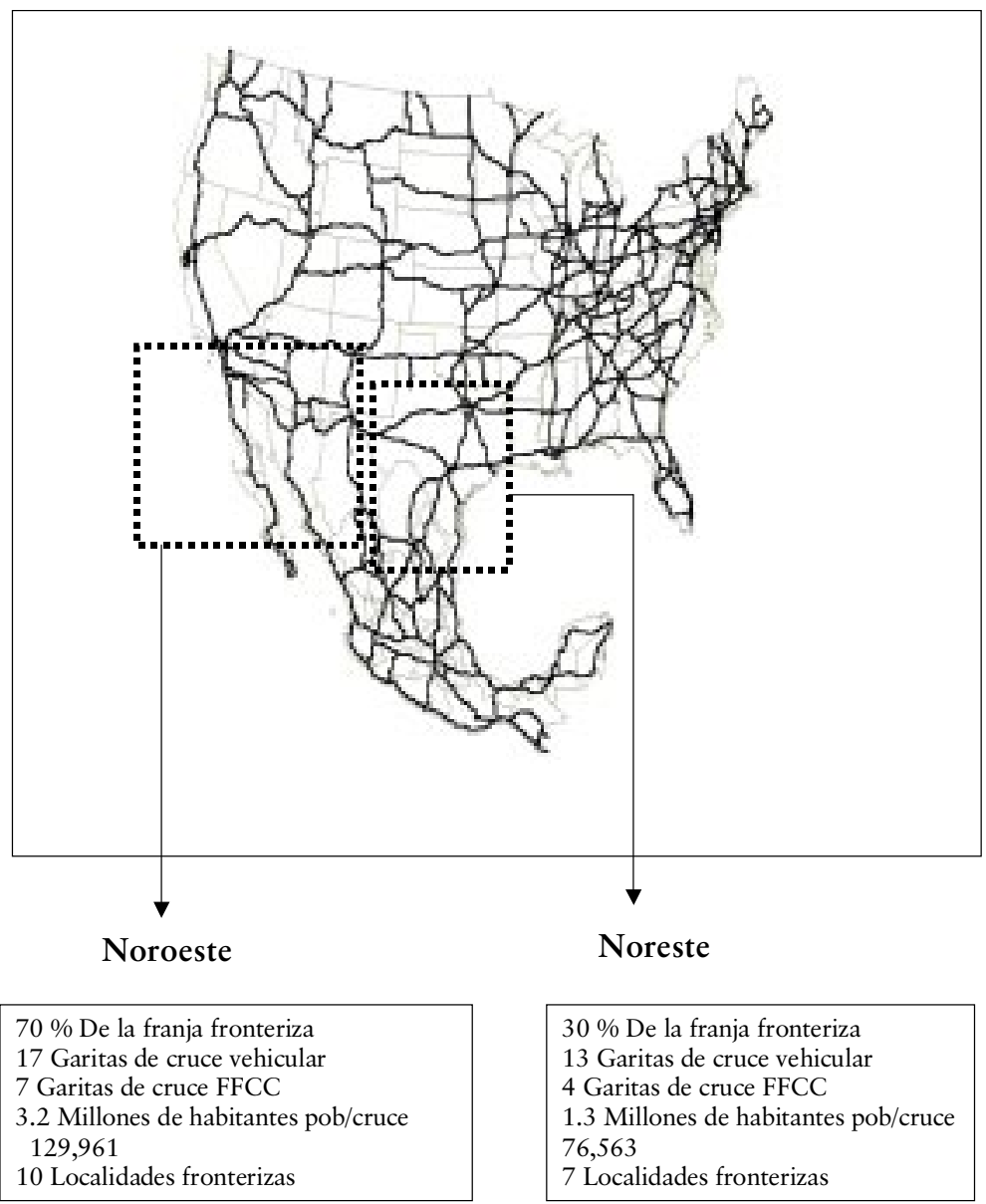

Fuente: Elaboración propia con base en información recabada e INEGI, Conteo de Población 1995.

bio urbano puede situarse a principios de la década de los setenta y no propiamente por el establecimiento de la IME, sino cuando se establece la paraestatal (luego privatizada) Minera Carbonífera Río Escondido (MICARE) y las plantas de Comisión Federal de Electricidad (CFE).

Existen dos tipos de circunstancias (o decisiones) que han logrado presionar por la utilización de recursos regionales diferenciados, originando las particularidades de la organización en 
los espacios habitados. Un tipo de circunstancias tiene que ver con la efectividad de acumulación de stock de capital que cada asentamiento permite o contiene su expansión por recursos existentes o futuros (indicadores de migración, territorio susceptible de incorporar a la red de producción y consumo, entre otros). Otro tipo de eventos son las decisiones de inversión que llevan a cabo instancias legitimadas o reconocidas (administración local, grupo de poseedores de capital, organismos políticos, etcétera), quienes son capaces de empujar hacia la efectiva acumulación y expansión económica presionando consuetudinariamente hacia el uso, eficiente o ineficaz, de mayores o mejores recursos.

La toma de decisiones centralizadas y alejadas de todo consenso social se refleja, a su vez, en pirámides de ingreso y recursos disponibles en el espacio urbano, que puede asimilarse a partir de la distribución de los espacios de recursos en los asentamientos. Esto ha logrado en algún sentido que una parte de la academia especializada en estudios urbanos se haya enfocado hacia el funcionamiento de la ciudad, es decir, el modo en el cual se organiza el intercambio de recursos en un espacio geográfico intervenido por las actividades del hombre. ${ }^{9}$

La traza tradicional que divide los espacios por medio de las administraciones políticas es rebasada por el empuje que el desplazamiento de la población y el incremento de las actividades económicas tienen en el entorno urbano de las localidades fronterizas. Cada uno de estos aspectos es receptivo a las condiciones que los actores locales fomentan o limitan. A reserva de documentar con mayores detalles que pudieran negarlo, en los asentamientos urbanos del noreste, a diferencia de los del noroeste, existen condiciones que hacen posible entenderlas como parte de un sistema de localidades que aprovechan el recurso de la distancia entre ellas, que les permite establecer y estructurar desplazamientos de población e inversión en actividades económicas, con menores costos y con mayores posibilidades de hacerlo eficientemente.

Del plano 2 se puede inferir la organización territorial de las localidades del noreste mayores de 50 mil habitantes, que para el año 2000 ha sumado más de 7 millones y medio de habitantes, cuya nodalidad se encuentra en el eje metropolitano SaltilloMonterrey. Este eje es alimentado a su vez por las localidades del

\footnotetext{
${ }^{9}$ A partir de esto se han derivado diferentes modelos de explicación como son el de Griffin y Ford, el de Gildersleeve, el de Hoffman, el de Arreola y Curtis, y el modelo específico fronterizo de Herzog. Mayores detalles consultar en Alarcón (1997) y Alarcón (1998).
} 
centro y norte de Coahuila, y la franja fronteriza de Tamaulipas, donde quizá el eje Victoria y la metrópoli Tampico-Madero observen intensidades de vinculación económica y urbana menores a los otros ejes, pero de relevancia decisiva como centro organizador de la política pública y puerto de embarque comercial. Agregado a esto se puede apreciar en el plano 3 la multiplicación de recursos viales y ferroviarios que desembocan en dos puertos. La redes vial y ferroviaria incluyen rutas norte-sur y este-oeste que se han ido expandiendo conforme aumenta la intensidad de los flujos.

\section{Plano 2}

Tamaño poblacional y ubicación geográfica de los municipios en las entidades de Coahuila, Nuevo León y Tamaulipas, 2000
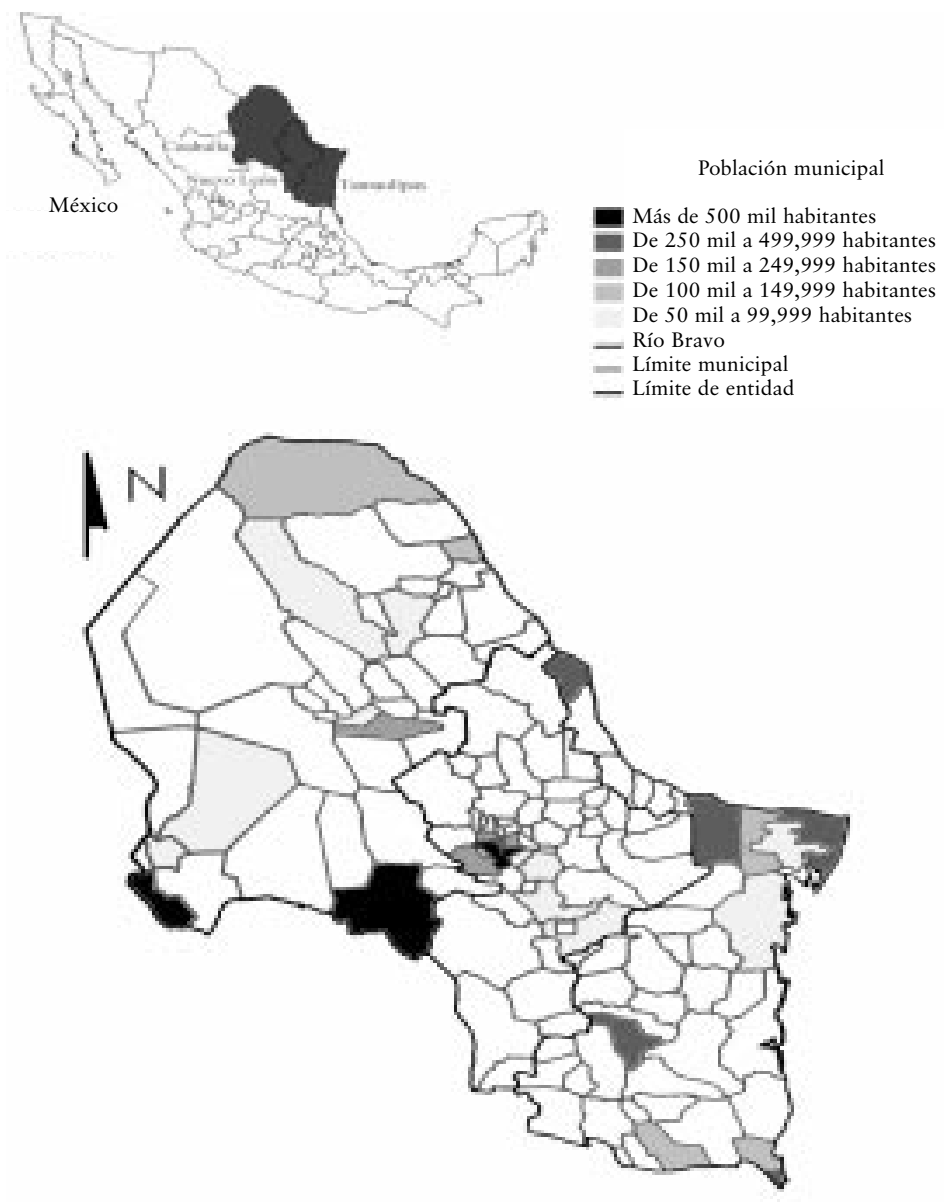

Fuente: Elaboración propia con base en INEGI, Resultados preliminares del Censo General de Población y Vivienda, 2000. 
Si observamos en el transcurso del tiempo el crecimiento de la población para algunos asentamientos seleccionados (véase el cuadro 1), podemos inferir tres procesos distintos que con probabilidad se observan en la mayoría de las localidades fronterizas: crecimiento lento y constante (Piedras Negras), crecimiento rápido en cierto periodo y decaimiento en el siguiente (Matamoros), y crecimiento lento en los inicios, rápido en un siguiente periodo y mediano, pero constante, en las últimas décadas (Reynosa). ${ }^{10}$ La diferencia de tales procesos indica, en primera instancia, que las condiciones temporales de crecimiento urbano son el resultado de anteriores decisiones de política que por consiguiente pueden modificarse a partir de otras políticas (Salazar, 1984: 9). Resulta relevante el hecho de que los patrones de expansión de la infraestructura correspondan a la intensificación de áreas altamente pobladas, lo que podría explicar la relación mutua que se guarda entre crecimiento poblacional y recursos invertidos en infraestructura.

\section{Plano 3 \\ Infraestructura vial, ferrocarrilera y portuaria en el noreste}
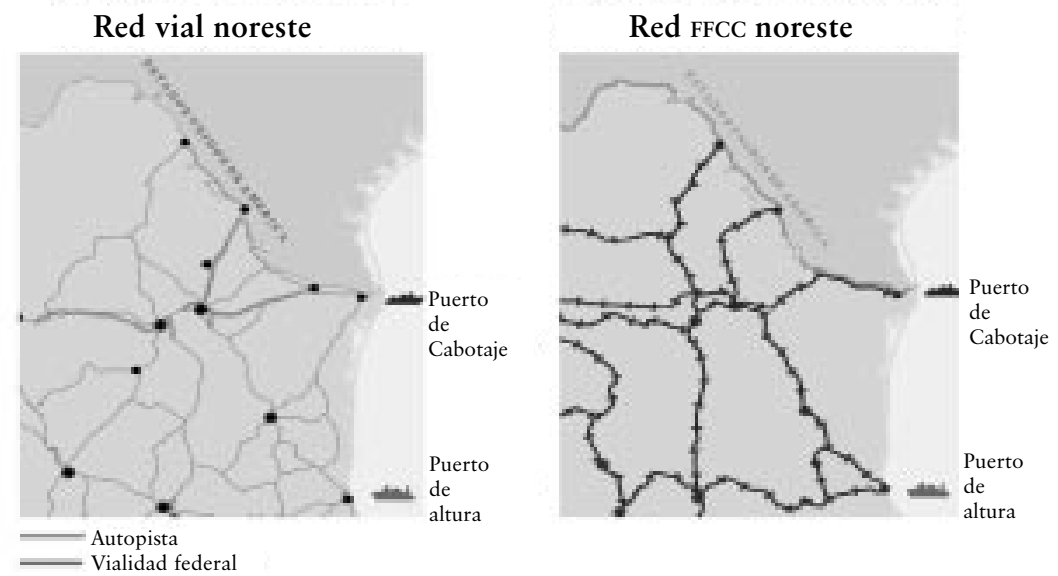

Fuente: Elaboración propia con base en INEGI, Mapas temáticos.

${ }^{10}$ La diferenciación del crecimiento poblacional en lento y rápido lo consideramos a partir de la media de crecimiento poblacional en los asentamientos fronterizos ( $C f r$. Gutiérrez y Vázquez, 1993). Incluso en Piedras Negras se observó una tasa decreciente de población (-0.6) en la década previa al establecimiento de MICARE y CFE, equilibrándose durante los setenta para luego disminuir. 


\section{Cuadro 1 \\ Población en varios años}

\begin{tabular}{|c|c|c|c|}
\hline Año & Piedras Negras & Matamoros & Reynosa \\
\hline 1850 & $300^{\mathrm{a}}$ & $11,233^{\mathrm{b}}$ & no disponible \\
1872 & no disponible & 13,740 & $3,724^{\mathrm{b}}$ \\
1900 & no disponible & $8,347^{\mathrm{b}}$ & $1,915^{\mathrm{b}}$ \\
1930 & $15,878^{\mathrm{d}}$ & 24,955 & 12,346 \\
1950 & 31,567 & $128,347^{\mathrm{a}}$ & 69,428 \\
2000 & $126,016^{\mathrm{c}}$ & $437,351^{\mathrm{c}}$ & $466,632^{\mathrm{c}}$ \\
\hline
\end{tabular}

aAño de su fundación "con menos de doscientos hombres... ( ) y 168 soldados" (Sánchez 1990: 11). ${ }^{\text {b }}$ (Alarcón, 1990). ${ }^{\text {c }}$ Población estimada por Gutiérrez y Vázquez (1993). ${ }^{\text {d }}$ (Unikel, 1978).

Fuente: Elaboración propia con base en las fuentes citadas y en el Censo General de Población y Vivienda 1930 y 1950.

Sin embargo, al introducirnos en la conformación intraurbana de las localidades se observa la relación dependiente de los vaivenes de los flujos de recursos (mercancías y servicios) que influyen en los cambios de su explotación y en la utilización del suelo urbano a través de la espacialidad del asentamiento, y donde es preciso entender el patrón productivo que expande la mancha urbana e intensifica las áreas de acuerdo con la intensificación del uso del suelo para actividades y población (que pueden significar cambios en las rutas o intensificación en el uso de las existentes ${ }^{11}$ ), con probables presiones que lleguen a rebasar la potencialidad del recurso, implicando su probable agotamiento o contaminación.

Se considera que los cambios urbanos en el noreste han establecido, hasta el momento, imperativos diversificados en las localidades que han visto aumentada la participación que tienen en crecimiento poblacional y económico. A esta diversidad le corresponde, empero, una constante que es la intensificación en el uso de los recursos, por lo que es posible encontrar patrones o modelos de desarrollo urbano altamente centralizados, puesto que dicha intensificación es resultado, a su vez, de patrones económicos que se desarrollan a medida que lo hacen las economías de aglomeración. También es preciso argumentar que este patrón es competido por los procesos de producción que basan su desempeño en la subcontratación, siendo la manufactura maquila-

${ }^{11}$ Alarcón (1998: 93) citando a Cerutti apunta que con el fortalecimiento de Nuevo Laredo como paso comercial sustituto de Matamoros puede interpretarse como el cambio de ruta, de Europa hacia los Estados Unidos. 
dora el sector que viene presionando, en el noreste, en ese sentido y que ha tenido importantes efectos en localidades fronterizas de rápido crecimiento en el noroeste. Estas consideraciones obligan a pensar en la existencia de imperativos urbanos, más allá de los económicos, cuya influencia ha sido escasamente incorporada a la planeación en la escala local y se convierte en la posibilidad mayor de controlar las presiones por urbanizar a que han estado sujetas las localidades fronterizas del norte de México, y en especial las del lado noreste.

\section{Conclusiones}

Para efectos de planeación urbana se han desarrollado una serie de recursos técnicos (sistemas de planeación e información geográfica), y se han ampliado los horizontes académicos hacia una adecuada comprensión de la problemática en las ciudades, la invención humana par excellence, de acuerdo con Claude LéviStrauss. Lo que se ofrece en este ensayo es la conveniencia de ir mas allá de los imperativos económicos e indagar el modo en el que se combinan con fenómenos exclusivos del acontecer local y regional, formando lo que denominamos imperativos urbanos.

En el documento se elaboraron dos aspectos: la pertinencia teórica del uso imperativos urbanos como un componente necesario en la búsqueda por entender la cuestión urbana, y su inicial documentación empírica a través de tres elementos: la infraestructura urbana, la planeación y las decisiones locales y regionales para el uso de recursos del hinterland. Entender la lógica del sistema urbano requiere considerar los alcances de los imperativos económicos, que generalmente son desdeñados en términos dinámicos en la planeación actual. Estos alimentan la intensificación y la expansión de áreas; sin embargo las circunstancias en las cuales se desarrolla la respuesta demográfica, salvo las perspectivas de probable expansión, no han sido suficientemente consideradas. Se tienen estimaciones del crecimiento de la población, pero no del modo en el que se distribuirá en la mancha urbana.

En ciudades de la frontera norte, y en particular en las del noreste, la constante ha sido, independientemente del tamaño, parámetros de segregación económica y fuertes tendencias hacia la excesiva densidad poblacional en relativamente pocas áreas de la mancha urbana de las ciudades, contrastando con la expansión urbana de las ciudades estadounidenses vecinas, no asimilado aún 
en las mexicanas. Es necesaria la documentación e investigación del modo en el cual se responde en las localidades ante la intervención económica del espacio; los condicionantes pueden ser la forma que presenta la producción, distribución y consumo capitalista en dos espacios intervenidos por actividades económicas semejantes (industria maquiladora, servicios financieros de exportación-importación, servicios administrativos y burocráticos, etcétera), dichas respuestas son definidas en Alonso (1998) como desenlaces territoriales.

Las diferencias cuantitativas que resultan de la presión que la economía tiene en el espacio urbano han significado en las localidades fronterizas dos claras diferencias cualitativas: la esfera de la intervención pública en la oferta de servicios urbanos en el que se inscribe la infraestructura para la convivencia (condiciones de la vivienda), discutida en buena medida en trabajos como los de Guillén (1990), Guillén et al. (1995 y 1997) Cabrero (1996a y 1996b), y Garrocho y Sobrino (1998), Schteingart (1989). La otra condición cualitativa del desempeño urbano se presenta por el empuje económico que da forma territorial a la división regional del trabajo que, a su vez, proporciona las condiciones de ingreso diferenciales para la cobertura cualitativa de las necesidades absolutas de protección y abrigo de la población.

Es probable que las próximas décadas se observe la consolidación de manifestaciones espaciales en la combinación de recursos locales que respondan a los imperativos económicos de la suburbanización intensiva del suelo, que de modo previsible -ceteris paribus- puede significar mayores indicadores de carencia y hacinamiento urbanos. Para evitar esto se requiere pensar la planeación como un instrumento de previsión y no de contención; que evite la especulación económica y política del uso del suelo, que ha venido conformando el mecanismo de incorporación de nuevas áreas a la mancha existente. La idea principal que guía este ensayo es la necesidad de orientar la planeación urbana desde la escala local con una visión más allá del cortoplacismo y virar hacia modelos de urbanización descentralizados que permitan responder a imperativos que empujan hacia la suburbanización. 
Los paradigmas de crecimiento urbano en las ciudades de la frontera de México tienen viejos esquemas de planeación y nuevas dinámicas económicas. La incompleta incorporación y empotramiento de ambos elementos en una visión de planeación, que aliente mecanismos de administración cotidiana de crecimiento urbano, desaprovechará las ventajas actuales de estructuración urbana que se presentan en algunas de ellas; complicará y desaprovechara las fuerzas de atracción metropolitana que existen como parte de metrópolis transfronterizas; y será rebasada por el empuje de los imperativos económicos sobre las necesidades sociales. Aunque las posibilidades son muchas porque las ciudades fronterizas han sido y continúan siendo receptoras de inversión local, nacional e internacional, los riesgos tienen también relevancia decisiva en las posibilidades de planear el crecimiento urbano que sea equiparable al desarrollo.

\section{Bibliografía}

Aguilar, Adrián G. et al. (1996), Las ciudades intermedias y el desarrollo regional en México, México, D. F., UNAM, Colmex y Conaculta, 403 pp.

Aguilar, Adrián G. y Francisco Rodríguez H. (1995), “Tendencias de desconcentración urbana en México, 1970-1990”, en Aguilar A. Guillermo, et al. (coordinadores), El desarrollo urbano de México a fines del siglo XX, Monterrey, N.L., Instituto de Estudios Urbanos de Nuevo León y Sociedad Mexicana de Demografía, pp. 75-100.

Aguilar, Ismael, Araceli Almaraz y Humberto Palomares (1998), Sistemas agroindustriales y desarrollo regional en la frontera norte de México, Reporte final de investigación financiado por la Fundación Colef.

Alarcón, Eduardo (1990), Evolución y dependencia en el noreste: las ciudades fronterizas de Tamaulipas, Tijuana, в.C., serie Cuadernos, El Colegio de la Frontera Norte, 58 pp.

Alarcón, Eduardo (1997), Interpretación de la estructura urbana de Laredo y Nuevo Laredo, Tijuana, B.C., El Colegio de la Frontera Norte, $96 \mathrm{pp}$. 
Alarcón, Eduardo (1998), La frontera de Tamaulipas y Texas: conformación de la estructura urbana, tesis de doctorado, unam, División de Estudios de Posgrado e Investigación, Facultad de Arquitectura.

Alegría, Tito (1994), “Condiciones espaciales de la pobreza urbana y una propuesta para su disminución”, en Frontera Norte especial núm. 1, Tijuana, B.C., El Colegio de la Frontera Norte, pp. 61-76.

Alonso, Jorge (1998), "La espacialidad social en el estudio de la industrialización: estructuras sin escalas, territorios sin sujetos”, en Región y Sociedad, El Colegio de Sonora, año IX, núm. 15 .

Bardhan, Pranab (1997), The Role of Governance in Economic Development. A political Economic Aproach, Centro de Estudios del Desarrollo de la OCDE, Paris, Francia, 94 pp.

Boltvinik, Julio (1994), "La satisfacción de las necesidades esenciales en México en los setenta y ochenta", en Pascual Moncayo y José Woldenberg (coordinadores), Desarrollo, desigualdad y medio ambiente, México, D.F., Cal y Arena.

Boltvinik, Julio (1996), “Diez tesis sobre la pobreza en México”, en Transición Mexicana, Ciclo de mesas redondas (memorias), Secretaría de Asuntos Estudiantiles, México, D.F., UNAM, pp. 203-214.

Burton, Elizabeth (2000), “The Compact City: Just or Just Compact? A Preliminary Analysis”, en Urban Studies, vol. 37, núm. 11, pp. 1969-2001.

Cabrero, Enrique (1996), Los dilemas de la modernización municipal: estudios sobre gestión hacendaria en municipios urbanos de México, México, D.F., Miguel Ángel Porrúa y CIDE.

Cabrero, Enrique et al. (1996), La nueva gestión municipal en México. Análisis de experiencias innovadoras en gobiernos locales, México, D.F., Miguel Ángel Porrúa. 
Cabrero, Enrique (1996a), Los dilemas de la modernización municipal: estudios sobre gestión hacendaria en municipios urbanos de México, México, D.F., Miguel Ángel Porrúa y CIDE.

Cabrero, Enrique et al. (1996b), La nueva gestión municipal en México. Análisis de experiencias innovadoras en gobiernos locales, México, D.F., Miguel Ángel Porrúa.

Camberos, Mario y Joaquín Bracamontes (1998), "Los niveles de bienestar en los noventa: un análisis comparativo entre la frontera norte de México y el país en su conjunto", en Roman y Vera Palacios (compiladores), La modernización contradictoria. Desarrollo humano, salud y ambiente en México, Guadalajara, Jalisco, U. de G. y CIAD, pp. 65-83.

Capello, Roberta (2000), “The City Network Paradigm: Measuring Urban Network Externalities”, en Urban Studies, vol. 37, núm. 11, pp. 1925-1945.

Castells, Manuel (1974), La cuestión urbana, México, Siglo XXI editores, $327 \mathrm{pp}$.

Dehghan, Farhad y Guillermo Vargas U. (1999), “Analysing Mexican Population Concentration: A Model with Empirical Evidence”, en Urban Studies, vol. 36, núm. 8, pp. 12691281.

Duranton, Gilles y Diego Puga (2000), "Diversity and Specialization in Cities: Why, Where and When Does it Matter?, en Urban Studies, vol. 37, núm. 3, pp. 533-555.

Edwing, Reid (1997), “Is Los Angeles-Style Sprawl Desirable?”, en Journal of the American Planning Association, vol. 63, núm. 1, American Planning Association, Chicago IL., pp. 107-126.

Faludi, Andreas (1998), "Why in Planning The Myth of the Framework Is Anything but That", en Philosophy of the Social Sciences, vol. 28, núm. 3., Sage Publications, pp. 381399. 
Garrocho, Carlos y Jaime Sobrino (1987), Sistemas de ciudades: fundamentos teóricos y operativos, Zinacantepec, Edo. de México, El Colegio Mexiquense.

Garza Gustavo (1996), Cincuenta anos de investigación urbana y regional en México, 1940-1991, México D.F., El Colegio de México, Centro de Estudios Demográficos y de Desarrollo Urbano, $325 \mathrm{pp}$.

Greenberg, Miriam (2000), "Branding Cities. A Social History of the Urban Lifestyle Magazine", en Urban Affairs Review, vol. 36, núm. 2, pp. 228-263.

Guillén, Tonatiuh (1990), "Servicios públicos y marginalidad social en la frontera norte", en Frontera Norte, Tijuana, B.c., vol. 2. núm. 4, pp. 95-120.

Guillén, Tonatiuh (coordinador) (1995), Municipios en transición. Actores sociales y nuevas politicas de gobierno, México, D.F., Fundación Friedrich Ebert Sriftung, 153 pp.

Guillén, Tonatiuh et al. (1997), La otra frontera norte de México. Sociedades y gobiernos de pequeñas dimensiones, México, D.F., Fundación Friedrich Ebert Sriftung, 221 pp.

Gutiérrez, Rodolfo y Gabriela Vázquez (1993), Proyecciones de población de municipios u condados, frontera México-Estados Unidos, Tijuana, B.c., (mimeo).

Hall, Peter (1992), Urban and Regional Planning, Londres y Nueva York, Routledge, $3^{\mathrm{a}}$ edn., $259 \mathrm{pp}$.

Harvey, David (1985), Urbanismo y desigualdad social, $3^{\mathrm{a}}$ edn., México, Siglo XXI editores, 340 pp.

Hernández, Enrique (1997), "Perspectivas del desarrollo regional en México frente a la globalización", en Economía, Teoría y Práctica, México, D.F., núm. 7, UAM, pp. 76-106.

Ingram, Gregory K. (1998), "Patterns of Metropolitan Development: What Have We Learned?”, en Urban Studies, vol. 35, núm. 7, pp. 1019-1035. 
Lefebvre, Henri (1991), The Production of Space, Cambridge, Massachusetts, Blackwell, 454 pp.

(1996), Writings on Cities, Oxford, UK, Cambridge, Mass., Blackwell, $250 \mathrm{pp}$.

Lo, Fu-chen y Peter J. Marcotullio (2000), "Globalization and Urban Transformations in the Asia-Pacific Region: A Review”, en Urban Studies, vol. 37, núm. 1, pp. 77-111.

Lowe Michelle S. (2000), "Britain's Regional Shoping Centres: New Urban Forms?", en Urban Studies, vol. 37, núm. 2, pp. 261-274.

Muheim, Philippe y David Freshwather (1999), “The Territorial Imperative", en Forum for Applied Research and Public Policy, pp. 90-95.

Pugh, Cedric (1997), "Poverty and Progress? Reflections on Housing and Urban Policies in Developing Countries, 1976-96”, en Urban Studies, vol. 34, núm. 10, pp. 15471597.

Robles, Vito Alessio (1979), Coahuila y Texas. Desde la consumación de la independencia hasta el tratado de paz de Guadalupe-Hidalgo, Biblioteca Porrúa S. A., núm. 73, 2ª edn., (2 tomos).

Rohe, William M., Richard E. Adams y Thomas A. Acury (2001), "Community Policing and Planning”, en APA Journal, vol. 67, núm. 1. pp. 78-90.

Salazar, Héctor (1984), La dinámica de crecimiento de las ciudades intermedias de México. Los casos de León, San Luis Potosí y Torreón, México, D.F., El Colegio de México, 110 pp.

Schteingart, Marta (1989), "Diez años de programas y políticas de vivienda en México”, en Gustavo Garza (compilador), Una década de planeación urbano regional, 1978-1988, México, D.F., El Colegio de México, pp. 211-231. 
Storper, Michael y Richard Walker (1989), The Capitalist Imperative, Territory, Technology, and Industrial Growth, Basil Backwell.

Storper, Michael y Robert Salais (1997), Worlds of Production. The action Frameworks of the Economy, Cambridge-London, Harvard University Press, 370 pp.

Zenteno, René (1993), Migración hacia la frontera norte de México: Tijuana, Baja California, Tijuana, B.C., serie Cuadernos 2, El Colegio de la Frontera Norte, 96 pp.

Enviado: 6 de noviembre de 2000 . Aceptado: 19 de febrero de 2001. 
